\title{
SCIDOC

\section{Infant Feeding Practices Implemented by HIV-Positive Mothers in South Africa}

Mbokane $\mathrm{AN}^{1}$, Ehlers $\mathrm{VJ}^{2 *}$, Roos $\mathrm{JH}^{2}$

${ }^{1}$ D Lit et Phil Graduate, Department of Health Studies, University of South Africa, South Africa.

${ }^{2}$ D Lit et Phil Professor, Department of Health Studies, University of South Africa, South Africa.

\section{Abstract}

Vertical transmission of HIV can occur during pregnancy, childbirth and breastfeeding. The South African Department of Health issues infant feeding guidelines which should be communicated to women attending prenatal clinics. These guidelines aim to balance a reduced risk of HIV infection with the best opportunity for the infants to survive and thrive. This study attempted to identify 200 conveniently selected HIV-positive mothers' infant feeding knowledge and practices by conducting structured face-to-face interviews in one province of South Africa.

Although all mothers had received health education about infant feeding options and most were knowledgeable about breast feeding, the guidelines issued by the Department of Health were not adhered to. Some mothers were erroneously informed to avoid breastfeeding and no demonstrations were done about preparing formula feeds. Some mothers mixed breast and formula feeding and some introduced solid foods before their babies were six months old.

Nurses working at prenatal and well-baby clinics, should be updated about revised official infant feeding guidelines to provide sustained relevant education about infant feeding to HIV-positive mothers. The outcomes of the South African programme to prevent vertical transmission of HIV could be jeopardised by mothers selecting inappropriate infant feeding options.

Keywords: Breastfeeding; Formula Feeding; Infant Feeding Options; HIV-Positive Mothers; Prevention of Vertical Transmission of HIV.

\section{Introduction}

Infant feeding is a critical part of the prevention of vertical transmission of HIV process [1], especially in resource poor countries. Thus the infant feeding options selected by HIVpositive mothers influence their babies' risk of HIV infection. Exclusive breastfeeding for the first six months has a limited risk of vertical transmission of HIV. Although exclusive formula feeding carries no such risk, formula feeding might be inaccessible to some HIV-positive mothers and/or unsafe for some babies. Formula feeding it could increase infant mortality rates due to diarrhoea and malnutrition in resource-limited countries $[1,2]$. Mixing breastfeeding with formula feeds increases the babies' risk of HIV infection. Giving solid foods to babies younger than six months of age could also increase this risk.

Infant feeding guidelines, as part of preventing vertical transmission of HIV, change as new information and challenges arise. These changes could cause confusion to health care providers and to mothers [2]. In 2008, South Africa's National Department of Health $(\mathrm{NDoH})[3]$ stipulated that an HIV-positive mother who opts for breastfeeding, should exclusively breastfeed her infant for six months only and then wean the baby abruptly.

However, the 2010 protocol [4] stipulated that HIV-positive pregnant women who choose to breastfeed, should do so exclusively for six months, commence with complementary feeds at six months, and continue with breastfeeding until the baby is 12 months old provided that either the mother or the baby takes anti-retroviral drugs.

The 2013 protocol [5] specifies that HIV-positive women who choose to breastfeed their infants, and who are not on lifelong anti-retroviral treatment, should be put on anti-viral drugs until

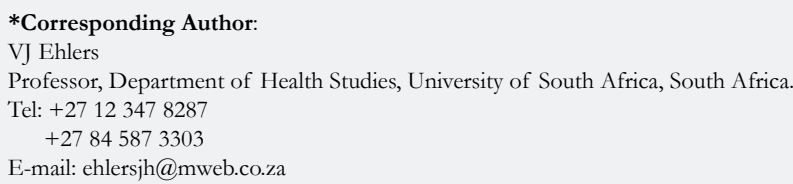

Copyright: Ehlers VJ ${ }^{\circ}$ 2016. This is an open-access article distributed under the terms of the Creative Commons Attribution License, which permits unrestricted use, distribution and reproduction in any medium, provided the original author and source are credited. 
one week after breastfeeding cessation. Since 2013 the South African government no longer provides formula, implying that mothers need to buy formula themselves [5].

The purpose of the current study was to identify HIV-positive mothers' infant feeding practices. The study's objectives were to identify whether the HIV-positive mothers were knowledgeable about available infant feeding options, which options they chose, who influenced their decisions and what was the HIV status of their babies at the age of six weeks. This information could then be incorporated into health education provided to HIV-positive pregnant women and mothers to reduce the risk of HIV transmission to their children after birth. South Africa spends a large proportion of its health budget on services to prevent vertical transmission of HIV, but the outcomes of this programme could be jeopardised by HIV-positive mothers who implement inappropriate infant feeding practices. This programme's outcomes could be significantly improved if HIV-positive mothers implemented appropriate infant feeding strategies.

\section{Materials and Methods}

A quantitative, descriptive, cross sectional survey was conducted in the Govan Mbeki area of the Mpumalanga Province of South Africa to identify HIV-positive mothers' infant feeding practices. The study population comprised all HIV-positive women who were using services in this area to prevent vertical transmission of HIV (known as prevention of mother-to-child or PMTCT services in South Africa). As no census of such women was available, convenience sampling was done. Women who used PMTCT services and who attended a well-baby clinic on the day when the researcher and research assistants collected data at that specific clinic were invited to participate in the study by being interviewed. Few women agreed to be interviewed as they feared disclosure of their HIV-positive status. By September 2011, data analysis commenced after 200 interviews had been conducted. A statistician assisted with the data analysis and interpretation.

The structured interview schedule requested biographic information and addressed the HIV-positive mothers' infant feeding practices and the relevance of this choice to their personal circumstances, persons who influenced the mother's selection of an infant feeding option, health education received about infant feeding options and the baby's HIV status at six weeks of age. The structured interview schedule was available in English and in isiZulu. The translation was approved by a bilingual expert. The instrument was pretested by conducting interviews with 10 mothers, excluded from the actual study. No problems were identified. The interviews were conducted by ten trained bilingual research assistants.

Two research experts accepted the structured interview schedule's face and criterion-related validity because all items requested information about the HIV-positive mothers' biographic details or about infant feeding practices in relation to South Africa's national infant feeding protocol. No inter-rater reliability coefficients could be established because every mother was only interviewed once, but the statistician detected no major discrepancies between instruments completed by different research assistants.
The University of South Africa's Higher Degrees Committee approved the research proposal (number 0771962 0) while the provincial health department and the manager of each participating clinic granted permission for data collection. The mothers were informed about the study when they attended a well-baby clinic. Those who were willing to be interviewed were consulted in private rooms, informed in detail about the purpose of the study and the nature of the individual interview, assured that neither their own nor their babies' treatment would be influenced by their decisions to participate or not to do so, and that no remuneration would be paid for participation. They could refuse to answer specific questions and could discontinue the interview at any stage without incurring any negative consequences whatsoever. They were also assured that no names would be used in any document and that all information would be treated strictly confidentially. The envisaged report would only contain facts and figures. Each mother who agreed to be interviewed signed a consent form which was kept in a sealed container while the anonymously completed interview schedules were kept in another sealed container. This ensured that no anonymously completed interview schedule could be linked to any signed consent form. Every research assistant signed a confidentiality agreement with the first author.

\section{Results and Discussions}

\section{Selected infant feeding options}

Out of the current study's 200 interviewed HIV-positive women, $36.5 \%(n=73)$ opted for formula feeding while $28.5 \% \quad(n=57)$ gave formula and solid foods to their babies. Only $15.5 \%(n=31)$ of the respondents practised exclusive breastfeeding, while $12.0 \%$ $(n=24)$ mixed breast milk, formula and solid foods, and $7.5 \%$ $(n=15)$ gave breast milk and solid foods.

The 200 respondents reportedly received the following types of advice pertaining to infant feeding options:

- $21.0 \%(\mathrm{n}=42)$ reported being told to breastfeed for six months only

- $15.5 \%(\mathrm{n}=31)$ were warned not to mix breast milk with formula and/or solid foods

- $30 \%(\mathrm{n}=60)$ had to choose between breastfeeding and formula feeding and

- $12.0 \%(\mathrm{n}=24)$ stated that they did not receive any information about infant feeding.

Out of 200 respondents in the current study, 89.5\% ( $n=179)$, made the final decision as to their babies' feeding methods but $10.5 \%(n=21)$ did not do so. In some cultures infant feeding is a family decision, especially in Sub Sahara Africa (SSA) where infant feeding is culturally embedded. A woman's decision has to be appropriate not only for the baby, but also for the community and her family (Aidsmap 2014b).

Though most respondents $(89.5 \% ; n=179)$, took their own infant feeding decisions, other people advised them, as shown in Table 1. The HIV counsellors had the greatest influence on the respondents' decisions $(36.5 \% ; \mathrm{n}=38)$, followed by nurses $(27.9 \%$; $\mathrm{n}=29)$, the respondents' mothers $(12.5 \% ; \mathrm{n}=13)$, and their sisters $(10.6 \%(\mathrm{n}=11)$. Their friends also had some influence $(7.7 \%$; 
Table 1. The person who influenced the decision taken on infant feeding $(n=104)$.

\begin{tabular}{|c|c|c|}
\hline $\begin{array}{c}\text { Persons Who Influenced The Respondents' } \\
\text { Infant Feeding Decisions }\end{array}$ & $\mathbf{n}$ & $\mathbf{\%}$ \\
\hline Nurses & 29 & 27.9 \\
\hline HIV counsellors & 38 & 36.5 \\
\hline Mothers & 13 & 12.5 \\
\hline Sisters & 11 & 10.6 \\
\hline Friends & 8 & 7.7 \\
\hline Partners & 5 & 4.8 \\
\hline Total & 104 & 100 \\
\hline
\end{tabular}

$n=8)$ whilst only $4.8 \%(n=5)$ of the respondents were influenced by their partners.

\section{Breastfeeding}

Out of 70 respondents who breast fed their babies, $60.0 \%(n=42)$ perceived breast milk to be safe, whilst $40.0 \%(n=28)$ regarded it as being unsafe, due to the presence of HIV in the breast milk. Some of the breastfeeding respondents $(51.4 \% ; n=36)$ would recommend breastfeeding to other HIV-positive women but $48.6 \%(n=34)$ would not do so.

Of the 70 respondents who breastfed their babies, $74.3 \%(n=52)$ had received advice, but $25.7 \%(\mathrm{n}=18)$ did not receive any advice from health care providers about safe breastfeeding for HIVpositive mothers.

Out of 52 respondents, who had received breastfeeding advice, $46.1 \%(n=24)$ were told to breastfeed for six months only, $34.6 \%$ $(\mathrm{n}=18)$ were taught not to mix breast milk with solids foods, whilst $15.4 \%(n=8)$ were advised to wash their hands before they breastfed their babies, and 3.9\% $(n=2)$ were told to check their nipples for cracks before breastfeeding.

The 70 breastfeeding respondents intended to breastfeed their babies for:

- $\quad$ Three months $(5.8 \% ; \mathrm{n}=12)$

- Six months $(67.1 \% ; n=45)$ and

- One year $(17.1 \% ; n=13)$.

No South African protocol mentions that HIV-positive women should refrain from breastfeeding as they could transmit HIV to their infants. The national protocols [3-5] specify that health care providers should supply correct information about the risk of HIV transmission in relation to different infant feeding options. The 2008 protocol [3] prescribed breastfeeding for six months with abrupt cessation. As the interviews were conducted until the end of September 2011, the healthcare workers should have been updated about the 2010 national protocol by that time.

Moland [6] states that mixed feeding is endemic in Sub Sahara Africa (SSA), as exclusive breastfeeding could be associated with being HIV-positive in many communities. Introducing solid foods before the baby is six months old, irritates the immature gut of the infant, increasing the baby's risk to HIV infection [6].
The national protocols [3-5] emphasise the importance of giving infant feeding education from the first time the pregnant woman visits the prenatal clinic and to continue with infant feeding counselling at every subsequent prenatal contact. The recommendation is that each pregnant woman is entitled to receive up to four infant feeding counselling sessions by the health care workers, lay counsellors and/or community care givers during her pregnancy.

Other factors might have influenced the mothers' decisions to breastfeed their babies despite their concerns about the safety of breast milk, such as the mothers' inability to pay for formula. Breast milk remains free, uncontaminated by impure water, suited for the baby's nutritional needs compared to formula feeding. Its properties do not irritate the baby's intestinal mucosa, and it promotes emotional wellbeing of the infant due to close physical contact with the mother during breastfeeding. Some respondents were concerned that if they did not breastfeed their babies, people might think that they were HIV-positive $(11.6 \%$; $n=20)$, while $12.1 \%(\mathrm{n}=21)$ mentioned that breast milk was always ready and $2.9 \%(\mathrm{n}=5)$ stated that their babies loved to be breastfed. The University of Bergen [9] highlighted that the risks of vertical transmission of HIV could be reduced during breastfeeding, but that the risk cannot be completely eliminated if the baby is breastfed.

Breastfeeding forms an integral part of the prevention of vertical transmission of HIV programme because of the presence of the HIV in the breast milk. If an HIV-positive woman chooses to breastfeed, the breastfeeding advice given, the content of the advice, and the duration of the breastfeeding period, could influence the risk of vertical transmission of HIV through breast milk.

The information outlined in Table 2, is in accordance with the replaced 2008 PMTCT protocol, which prescribed that an HIVpositive woman should breastfeed for six months only [3]. The 2010 national protocol [3] recommended breastfeeding the baby for one year. Nyamanhindi [10] stated that infant feeding information tends to involve the community, and it might take some time for the implementers of the prevention of vertical transmission of HIV programme to replace old information with new information.

Only $17.1 \%(n=13)$ of the 70 breastfeeding respondents intended breastfeeding their babies for one year, meeting the stipulated one year duration of breastfeeding in terms of the national 
Table 2. Advice provided about safe breastfeeding practices $(n=52)$.

\begin{tabular}{|c|c|c|}
\hline $\begin{array}{c}\text { Safe Breastfeeding Practices Giiven To Hiv-Posi- } \\
\text { tive Breastfeeding MotherS }\end{array}$ & Frequency & Percentage \\
\hline Breastfeed for 6 months only & 24 & 46.1 \\
\hline Do not give water, juice or solids with the breast milk & 18 & 34.6 \\
\hline Wash your hands before breastfeeding & 8 & 15.4 \\
\hline Check your breast for cracks before breastfeeding & 2 & 3.9 \\
\hline Total & 52 & 100 \\
\hline
\end{tabular}

protocol [4]. No reasons could be derived from the available data for women intending to breastfeed their babies for six months $(67.1 \% ; n=45)$ or for three months $(15.8 \% ; n=12)$, implying that $82.9 \%(\mathrm{n}=57)$ of the breastfeeding women in the current study, would not breastfeed their babies for the recommended 12 months. This finding was unexpected as early cessation of breastfeeding is foreign to most African countries [11].

When the breastfeeding mothers were asked as to why they chose to breastfeed their babies, the following benefits of breastfeeding were mentioned that breast mild.

- $\quad$ protects babies against diseases $(34.2 \% ; n=24)$

- is healthy for the babies $17.4 \%(\mathrm{n}=12)$ and

- $\quad$ it is free $(11.4 \% ; n=8)$.

Breast milk contains whey proteins that are easily absorbed by the baby's gut [10]. It has no allergens that could irritate the baby's intestinal canal. Breast milk also contains lipase and enzymes that aid fat digestion.

\section{Formula feeding}

There is no chance of vertical transmission of HIV through formula feeds. Some resource rich countries have effectively implemented formula feeding as a PMTCT strategy, which has helped to reduce the risk of vertical transmission of HIV to $2 \%$. In resource poor countries, formula feeding could result in diarrhoea, malnutrition and babies' deaths [12].

According to South Africa's national protocol [3] a woman choosing to formula feed her baby should ensure that formula feeding is affordable, feasible, acceptable, suitable and sustainable (AFASS) for her individual circumstances.

When the respondents were asked about the infant feeding method they would have chosen if they were HIV negative, $86.5 \%(n=173)$ stated that they would have breastfed their babies, and $13.5 \%(n=27)$ said they would have formula fed anyway.

However, only $35.0 \%(n=70)$ of these HIV-positive women did breastfeed their babies (although merely $15.5 \%$ or 31 respondents practised exclusive breastfeeding) while $65.0 \%(n=130)$ did not breastfeed their babies at all. These respondents might have perceived themselves as being vulnerable to transmitting HIV to their babies through breastfeeding and they ended up formula feeding.

In total 154 of the current study's 200 respondents gave formula feeds to their babies, because out of the 70 respondents who breastfed their babies, 24 gave breast milk and formula feeds. According to the national protocol [4], women who choose to breastfeed must exclusively breast feed for six months, and introduce solid foods from six months. She must continue to breast feed for 12 months. Those who choose to formula feed their babies, must give only formula for six months and introduce complementary feeds at six months.

Only $59.8 \%(\mathrm{n}=92)$ of the 154 respondents received infant formula from clinics and $40.2 \% \quad(n=62)$ bought their own formula. The 2010 protocol [4] prescribes that women who choose the formula infant feeding option should be provided with formula according till the infant was six months old, which was the case while the interviews were being conducted for the current study. During the interviews, those respondents who bought their own formula, were asked whether they could afford to buy formula. As many as $40.3 \%(n=25)$ of these women could not afford to buy the formula and $82.5 \%(n=51)$ used less than R500 (approximately USD33.34) to buy their monthly supplies of formula, which might have been insufficient for babies aged six months and older.

Respondents were asked if they could read and understand the instructions on the infant formula containers. As many as $28.6 \%$ $(n=44)$ of the 154 respondents could not read and/or understand the instructions, and $71.4 \%(\mathrm{n}=110)$ could do so, possibly because the majority $(81.5 \% ; n=163)$ of these respondents had acquired some secondary school education. Out of 154 respondents who selected the formula feeding option, $60.4 \%(n=93)$ reportedly obtained information to prepare these feeds from the infant formula containers, $24.7 \% \quad(n=38)$ were helped by family members, and $14.9 \%(n=23)$ obtained information from the HIV counsellors. (Although 130 respondents used exclusive formula feeding, 24 of the women who breastfed their babies also gave infant formula to their babies, explaining why these calculations were based on a total of 154 respondents who used formula feeding for their babies).

The national protocol [4] prescribes that infant feeding should form part of the post-test counselling of HIV-positive women. Infant formula preparation has to be demonstrated by the health workers, and the education should include the volume, frequency, dangers associated with formula feeding, and how to cup feed the baby. Bottle feeding should be discouraged as it is associated with poor hygiene of infant bottles, and babies become predisposed to diarrhoea. The content of this education has to be repeated at every visit to the clinic.

Respondents obtained water for preparing the babies' formula feeds from communal taps $(33.1 \% ; \mathrm{n}=51)$ and from taps in their 
houses $(66.9 \% ; n=103)$. Thus all 154 respondents who selected the infant formula feeding option had access to tap water.

A total of $73.4 \%(n=113)$ of the respondents reported that their family members were content with their choice to formula feed their babies and 26.6\% $(n=41)$ said this was not the case. Of 113 respondents who stated that their families were content with infant formula as a means of infant feeding, 58.6\% $(n=68)$ stated that their families knew about their HIV-positive status, 15.5\% $(\mathrm{n}=18)$ were working and not always available to breastfeed, $11.2 \%(n=13)$ could not exclusively breastfeed, and $14.7 \%(n=17)$ said it was their choice to make, their families had no say.

\section{Solid foods and mixed feeding}

Out of the 96 babies who had been given solid foods, only $35.4 \%$ $(n=34)$ commenced with solid foods at the age of six months, while others did so at younger ages. According to Aidsmap [7] and Avert [8], the early introduction of solid foods is the norm in many African countries. This poses challenges because SSA has many HIV-positive women who should implement practices to prevent the vertical transmission of HIV. The early introduction of solid foods could increase the HIV incidence among babies due to the increased permeability of their immature digestive systems making them susceptible to HIV infection. The risk of vertical transmission of HIV was increased, by the introduction of solid foods before the babies were six months old, for $19.5 \%$ $(n=39)$ of the infants, since solids were given concurrently with the HIV-positive mothers' breast milk.

\section{Challenges identified with regards to infant feeding}

The identified challenges were categorised as those that were respondent-related and those that were health provider-related. Respondent-related challenges included that:

- $11.0 \%(\mathrm{n}=22)$ of the respondents mixed fed their babies with breast milk, formula feeds and/or solids.

- $10.5 \%(n=21)$ of the women felt it was not their ultimate decision on how their infants were fed.

- $46.5 \%(n=93)$ of the infants were given solid foods, and $64.5 \%(n=60)$ had been introduced to solids before they were six months old.

- $11.5 \%(n=11)$ out of 93 respondents who used mixed feeding methods, introduced solids at birth.

- out of $35.0 \%(n=70)$ of the respondents who breastfed their babies, $60.0 \%(n=42)$ did not perceive it to be safe, because breast milk could transmit HIV.

Health service/provider-related infant feeding challenges included that:

- $12.0 \%(\mathrm{n}=24)$ of the respondents were reportedly not given any infant feeding information, and $16.0 \%(n=32)$ reported been told not to breastfeed, as they would transmit HIV to their babies. According to the national protocol [4] no HIVpositive mother should be advised to avoid breastfeeding because she might transmit HIV to her baby.

- No demonstrations were conducted at the clinics on how to prepare the formula feeds. According to the national protocol $[4,5]$ formula feeding women should receive practical support on how to prepare infant feeds using clean surfaces, tools and clean hands. Out of 130 respondents, 60.0\% $(n=93)$ mentioned that the infant formula container was their source of information, and $25.0 \%(n=38)$ mentioned being helped by members of their families because they did not obtain this information from health care providers.

- $22.3 \%(n=15)$ of the respondents were not informed about safe breastfeeding practices.

\section{The HIV status of the respondents' children}

Children whose mothers were on the prevention of vertical transmission of HIV programme during pregnancy, undergo PCR tests to determine each child's HIV status at the visit to the clinic when the child is six weeks old.

When the HIV status of the respondents' children were assessed using the PCR results, $91.0 \%(n=182)$ were found to be HIV negative, $9.0 \%(n=18)$ were HIV-positive.

According to the national protocol4, a breastfed baby should be re-tested for HIV six weeks after the cessation of breastfeeding. As the PCR tests were done when the babies were six weeks old, when $35.0 \%(n=70)$ were still being breast-fed, more babies might have tested HIV-positive six weeks after breastfeeding had been discontinued. However, this information could not be obtained during the data collection phase of this study

\section{Limitations of the study}

This current study's structured interviews were conducted with $200 \mathrm{HIV}$-positive mothers during 2011, soon after the introduction of the 2010 infant feeding protocol [4]. Thus some respondents might have remembered the information of the 2008 protocol3.

The study was conducted in one area of one province of South Africa. The findings can only be generalised to other parts of the country after similar studies had been conducted in different areas.

Interviews were conducted with 200 conveniently selected HIVpositive mothers willing to be interviewed. Women who refused to be interviewed might have had different levels of knowledge about infant feeding and might have implemented different feeding strategies.

\section{Conclusions}

Most HIV-positive mothers did not follow the national protocol [4] of breastfeeding their babies exclusively for six months, introducing supplementary feeds at six months of age but continuing with breastfeeding till the baby is 12 months old. Most infants received formula feeds, sometimes concurrently with breast milk and/or solid foods which were introduced soon after birth. These infant feeding practices might have increased the babies' risk of vertical HIV infection, impacting negatively on the outcomes of the prevention of vertical transmission of HIV programme in South Africa.

The health care providers did not inform all HIV-positive women about the national protocol, about breastfeeding practices nor about formula feeding and never demonstrated the preparation 
of formula feeds.

\section{Recommendations}

Health care workers, including health counsellors, should be updated about the current national infant feeding protocol [5] and share appropriate information with HIV-positive women at every encounter. Health education provided to HIV-positive women should be monitored for accuracy. Regular follow-up investigations should be conducted and identified infant feedingrelated challenges should be addressed. The outcomes of the national programme to prevent vertical transmission of HIV are affected by HIV-positive mothers' infant feeding practices.

\section{Acknowledgement and Declarations}

The authors thank the authorities that granted permission for the study to be conducted, the research assistants who interviewed the respondents and the HIV-positive mothers who shared their experiences and perceptions with the researchers.

The paper is based on part of the research conducted for the first author's doctoral thesis. The other two authors were the supervisors of the study.

No funds were received for conducting this study. The authors declare that they encountered no conflict of interest in conducting the study and in writing the paper as no person and no institution influenced them to do so in any specific manner.

\section{References}

[1]. World Health Organization (2013) Consolidated guidelines on the use of antiretroviral drugs for treating and preventing HIV infection: Recommendations for a public health approach. 272 .

[2]. United Nations Children's Fund (2012) Breast feeding and HIV transmission: HIV and Infant Feeding.

[3]. National Department of Health (2008) Policy and guidelines for the implementation of the PMTCT programme.Government Printer, Pretoria. 1-84.

[4]. National Department of Health (2010) Policy and guidelines for the implementation of the PMTCT programme. Government Printer, Pretoria.

[5]. National Department of Health (2013) The South African antiretroviral Treatment Guidelines. Government Printer, Pretoria.1-21.

[6]. Moland KM, Van Esterik P, Sellen DW, De Paoli MM, Leshabari SC, et al., (2010) Ways ahead: protecting, promoting and supporting breastfeeding in the context of HIV .Int Breast Feeding J 5: 12.

[7]. Aidsmap (2014) Mixed versus exclusive breastfeeding.

[8]. Avert (2014) HIV and breast feeding.

[9]. University of Bergen (2014) More children of HIV positive mothers saved.

[10]. N yamanhindi R (2014) Exclusive breast feeding protects newborns from HIV/AIDS.

[11]. Blystad A, Van Esterik P, De Paoli MM, Sellen DW, Leshabari SC, et al., (2010) Reflections on global policy documents and the WHO's infant feeding guidelines, lesson learnt. 5:18. DOI: 10.1186/1746-4358-5-18.

[12]. World Health Organization (2011) New guidance on prevention of mother to child transmission of HIV and infant feeding in the context of HIV. 